\title{
Generalized Fractional Nonlinear Birth Processes
}

\author{
Mohsen Alipour • Luisa Beghin · Davood Rostamy
}

Received: 4 March 2013 / Revised: 12 August 2013 /

Accepted: 15 August 2013 / Published online: 31 August 2013

(c) Springer Science+Business Media New York 2013

\begin{abstract}
We consider here generalized fractional versions of the differencedifferential equation governing the classical nonlinear birth process. Orsingher and Polito (Bernoulli 16(3):858-881, 2010) defined a fractional birth process by replacing, in its governing equation, the first order time derivative with the Caputo fractional derivative of order $v \in(0,1]$. We study here a further generalization, obtained by adding in the equation some extra terms; as we shall see, this makes the expression of its solution much more complicated. Moreover we consider also the case $v \in(1,+\infty)$, as well as $v \in(0,1]$, using correspondingly two different definitions of fractional derivative: we apply the fractional Caputo derivative and the rightsided fractional Riemann-Liouville derivative on $\mathbb{R}_{+}$, for $v \in(0,1]$ and $v \in(1,+\infty)$, respectively. For the two cases, we obtain the exact solutions and prove that they coincide with the distribution of some subordinated stochastic processes, whose random time argument is represented by a stable subordinator (for $v \in(1,+\infty)$ ) or its inverse (for $v \in(0,1])$.
\end{abstract}

Keywords Generalized fractional birth process • Fractional Caputo derivative • Fractional Riemann-Liouville derivative • Mittag-Leffler functions •

Stable subordinator

\footnotetext{
M. Alipour

Faculty of Basic Science, Babol University of Technology, P.O. Box 47148-71167, Babol, Iran e-mail: m.alipour2323@gmail.com

L. Beghin $(\bowtie)$

Department of Statistical Sciences, Sapienza University of Rome, Rome, Italy

e-mail: luisa.beghin@uniroma1.it

D. Rostamy

Department of Mathematics, Imam Khomeini International University, P.O. Box 34149-16818, Qazvin, Iran

e-mail: rostamy@khayam.ut.ac.ir
} 
AMS 2010 Subject Classifications $60 \mathrm{G} 52 \cdot 34 \mathrm{~A} 08 \cdot 33 \mathrm{E} 12 \cdot 26 \mathrm{~A} 33$

\section{Introduction and Preliminaries}

We consider here the following Cauchy problem

$$
\left\{\begin{array}{l}
\frac{d^{v}}{d t^{v}} P_{1}^{v}(t)=-\lambda_{1} P_{1}^{v}(t) \\
\frac{d^{v}}{d t^{v}} P_{k}^{v}(t)=-\lambda_{k} P_{k}^{v}(t)+\sum_{i=1}^{k-1} \lambda_{i} P_{i}^{v}(t), \quad k=2,3, \ldots,
\end{array}\right.
$$

for $t \geq 0, v>0$, with initial conditions

$$
P_{k}^{v}(0)=\left\{\begin{array}{ll}
1 & k=1, \\
0 & k=2,3, \ldots
\end{array},\right.
$$

where $\lambda_{j} \in \mathbb{R}, j=1,2, \ldots$.

We will obtain exact solutions to Eqs. 1 and 2 in both cases $v \in(0,1]$ and $v \in$ $(1,+\infty)$; in the first case they will be expressed in terms of finite sums of the MittagLeffler functions and in the second case as finite sums of exponentials.

While the first equation in Eq. 1 is the so-called fractional relaxation equation (see e.g. Uchaikin 2002; Beghin 2012), we can call the second equation in Eq. 1 a generalized fractional birth equation (see e.g. Orsingher and Polito 2010, 2011, 2013; Cahoy and Polito 2012). Indeed, in Orsingher and Polito (2010) and later in Orsingher and Polito (2013), the authors analyze the following Cauchy problem

$$
\frac{d^{v}}{d t^{v}} P_{k}^{v}(t)=-\lambda_{k} P_{k}^{v}(t)+\lambda_{k-1} P_{k-1}^{v}(t), \quad k \geq 1, \quad v \in(0,1],
$$

(where $P_{k}^{v}(0)=1_{[k=1]}$ and $\frac{d^{v}}{d t^{v}}$ denotes the Caputo fractional derivative) and define the corresponding process "fractional birth process". Equation 3 is formally connected to Eq. 1, even though it cannot be obtained as a particular case, since, by assumption, it is $\lambda_{j} \neq \lambda_{i}$, for $j \neq i$, in Eq. 1. The extra terms in the second equation of Eq. 1 makes the expression of its solution much more complicated.

We will analyze the problem (1)-(2) for any value of $v$ : we need to distinguish the two cases $v \in(0,1]$ and $v \in(1,+\infty)$, because we use correspondingly two different definitions of fractional derivative (see, for example, Kilbas et al. 2006):

1. For $v \in(0,1]$, we apply the fractional Caputo derivative of order $v$, which is defined as follows:

$$
{ }_{C} D_{0+, t}^{v} f(t):=\frac{1}{\Gamma(1-v)} \int_{0}^{t}(t-s)^{-v} \frac{d}{d s} f(s) d s, \quad t>0 .
$$

2. For $v \in(1,+\infty)$, we use the right-sided fractional Riemann-Liouville derivative on $\mathbb{R}_{+}$of order $v$, which is defined as follows:

$$
{ }_{R L} D_{-, t}^{v} f(t):=\frac{1}{\Gamma(m-v)}\left(-\frac{d}{d t}\right)^{m} \int_{t}^{+\infty} \frac{f(s)}{(s-t)^{v-m+1}} d s, \quad t>0,
$$

where $m=\lceil v\rceil$. 
Note that, for $v=1$, we have ${ }_{C} D_{0+, t}^{v}=\frac{d}{d t}$ and ${ }_{R L} D_{-, t}^{v}=-\frac{d}{d t}$, where $\frac{d}{d t}$ is the standard derivative. Throughout this paper we always use the symbol $\frac{d^{v}}{d t^{v}}$ and we set

$$
\frac{d^{v}}{d t^{v}}:=\left\{\begin{array}{ll}
{ }_{C} D_{0+, t}^{v} & v \in(0,1], \\
{ }_{-}{ }_{R L} D_{-, t}^{v} & v \in(1,+\infty)
\end{array} .\right.
$$

The study of fractional generalizations of classical differential equations has attracted a lot of attention over the last decades: see, for example, Mainardi (1996), Angulo et al. (2000) and Orsingher and Beghin (2009) for fractional diffusions; Mainardi et al. (2004), Beghin and Orsingher (2009) and Meerschaert et al. (2011) for equations related to fractional Poisson and renewal processes.

Finally we prove that the solution to Eq. 1 can be expressed as the distribution of the stochastic process defined as

$$
N_{v}(t):=N_{1}\left(H_{v}(t)\right)
$$

where $N_{1}(t), t \geq 0$ is the process governed by Eq. 1 with $v=1$, and

$$
H_{v}(t):=\left\{\begin{array}{ll}
L^{v}(t), & v \in(0,1) \\
A^{\frac{1}{v}}(t), & v \in(1,+\infty)
\end{array}, \quad t \geq 0,\right.
$$

with $H_{v}(t):=t$ for $v=1$. The processes $A^{\frac{1}{v}}(t)$ and $L^{v}(t)$ in Eq. 4 are respectively a stable subordinator of index $\frac{1}{v}$ and the inverse of a stable subordinator of index $v$ (see Theorem 5 below for their exact definitions).

Due to the different properties of the sample paths between the processes given in Eq. 4, the two forms of fractional birth processes presented here display a very different behavior, in the two cases $v \in(0,1]$ and $v \in(1,+\infty)$. Indeed in the first case the process can perform only unitary jumps in an interval of infinitesimal length (as in the standard case $v=1$ ), while in the latter the number of jumps can be of any integer size.

\section{Main Results}

We start by considering the case where $v \in(0,1]$ and deriving the Laplace transform of the solution to Eqs. 1 and 2. We use the following notation:

$$
L[f(t) ; \mu]:=\int_{0}^{\infty} e^{-\mu t} f(t) d t
$$

Theorem 1 For the fractional equations

$$
\left\{\begin{array}{l}
\frac{d^{v}}{d t^{v}} P_{1}^{v}(t)=-\lambda_{1} P_{1}^{v}(t) \\
\frac{d^{v}}{d t^{v}} P_{k}^{v}(t)=-\lambda_{k} P_{k}^{v}(t)+\sum_{i=1}^{k-1} \lambda_{i} P_{i}^{v}(t), \quad k=2,3, \ldots,
\end{array} \quad t>0, \quad 0<v \leq 1,\right.
$$


with initial conditions

$$
P_{k}^{v}(0)=\left\{\begin{array}{ll}
1 & k=1 \\
0 & k=2,3, \ldots
\end{array},\right.
$$

for $\lambda_{i} \in \mathbb{R}(i=1,2, \ldots)$, we have

$$
L\left[P_{k}^{v}(t) ; \mu\right]= \begin{cases}\frac{\mu^{v-1}}{\mu^{v}+\lambda_{1}}, & k=1 \\ \frac{\lambda_{1} \mu^{v-1}}{\left(\mu^{v}+\lambda_{1}\right)\left(\mu^{v}+\lambda_{2}\right)}, & k=2 \\ \frac{\lambda_{1} \mu^{v-1}}{\left(\mu^{v}+\lambda_{1}\right)\left(\mu^{v}+\lambda_{k}\right)} & \sum_{\left(q_{2}, \ldots, q_{k-1}\right) \in A_{k}} \prod_{i=2}^{k-1}\left(\frac{\lambda_{i}}{\mu^{v}+\lambda_{i}}\right)^{q_{i}}, k=3,4, \ldots\end{cases}
$$

where

$$
A_{k}=\left\{\left(q_{2}, q_{2}, \ldots, q_{k-1}\right) \mid q_{i} \in\{0,1\}, i=2, \ldots, k-1\right\}
$$

Proof We prove the result (7) by induction.

For $k=1$,

$$
\frac{d^{v}}{d t^{v}} P_{1}^{v}(t)=-\lambda_{1} P_{1}^{v}(t), \quad P_{1}^{v}(0)=1,
$$

by the Laplace transform we get

$$
\mu^{v} L\left[P_{1}^{v}(t) ; \mu\right]-\mu^{v-1} P_{1}^{v}(0)=-\lambda_{1} L\left[P_{1}^{v}(t) ; \mu\right], \quad \Rightarrow \quad L\left[P_{1}^{v}(t) ; \mu\right]=\frac{\mu^{v-1}}{\mu^{v}+\lambda_{1}} .
$$

For $k=2$, Eq. 5 becomes

$$
\frac{d^{v}}{d t^{v}} P_{2}^{v}(t)=-\lambda_{2} P_{2}^{v}(t)+\lambda_{1} P_{1}^{v}(t), \quad P_{2}^{v}(0)=0 .
$$

By taking the Laplace transform, we have

$$
\begin{aligned}
\mu^{v} L\left[P_{2}^{v}(t) ; \mu\right]-\mu^{v-1} P_{2}^{v}(0) & =-\lambda_{2} L\left[P_{2}^{v}(t) ; \mu\right]+\lambda_{1} \frac{\mu^{v-1}}{\mu^{v}+\lambda_{1}}, \\
& \Rightarrow L\left[P_{2}^{v}(t) ; \mu\right]=\frac{\lambda_{1} \mu^{v-1}}{\left(\mu^{v}+\lambda_{1}\right)\left(\mu^{v}+\lambda_{2}\right)} .
\end{aligned}
$$

For $k=3$, Eq. 5 becomes

$$
\frac{d^{v}}{d t^{v}} P_{3}^{v}(t)=-\lambda_{3} P_{3}^{v}(t)+\lambda_{2} P_{2}^{v}(t)+\lambda_{1} P_{1}^{v}(t), \quad P_{3}^{v}(0)=0 .
$$

By taking the Laplace transform, we get

$$
\begin{aligned}
\mu^{v} L\left[P_{3}^{v}(t) ; \mu\right]-\mu^{v-1} P_{3}^{v}(0)= & -\lambda_{3} L\left[P_{3}^{v}(t) ; \mu\right] \\
& +\lambda_{2} \frac{\lambda_{1} \mu^{v-1}}{\left(\mu^{v}+\lambda_{1}\right)\left(\mu^{v}+\lambda_{2}\right)}+\lambda_{1} \frac{\mu^{v-1}}{\left(\mu^{v}+\lambda_{1}\right)} .
\end{aligned}
$$


Therefore, we obtain

$$
\begin{aligned}
L\left[P_{3}^{v}(t) ; \mu\right] & =\lambda_{2} \frac{\lambda_{1} \mu^{v-1}}{\left(\mu^{v}+\lambda_{1}\right)\left(\mu^{v}+\lambda_{2}\right)\left(\mu^{v}+\lambda_{3}\right)}+\lambda_{1} \frac{\mu^{v-1}}{\left(\mu^{v}+\lambda_{1}\right)\left(\mu^{v}+\lambda_{3}\right)} \\
& =\frac{\lambda_{1} \mu^{v-1}}{\left(\mu^{v}+\lambda_{1}\right)\left(\mu^{v}+\lambda_{3}\right)}\left(\frac{\lambda_{2}}{\mu^{v}+\lambda_{2}}+1\right) .
\end{aligned}
$$

Similarly, for $k=4$, we get

$$
\begin{aligned}
L\left[P_{4}^{v}(t) ; \mu\right]= & \frac{\lambda_{1} \mu^{v-1}}{\left(\mu^{v}+\lambda_{1}\right)\left(\mu^{v}+\lambda_{4}\right)} \\
& \times\left(\frac{\lambda_{2} \lambda_{3}}{\left(\mu^{v}+\lambda_{2}\right)\left(\mu^{v}+\lambda_{3}\right)}+\frac{\lambda_{2}}{\left(\mu^{v}+\lambda_{2}\right)}+\frac{\lambda_{3}}{\left(\mu^{v}+\lambda_{3}\right)}+1\right) .
\end{aligned}
$$

Then, we can see that Eq. 7 holds for $k=1,2,3,4$.

Now, we suppose that Eq. 7 holds for $L\left[P_{i}^{v}(t) ; \mu\right], i=1, \ldots, k-1$. Then we will prove that Eq. 7 is true for $L\left[P_{k}^{v}(t) ; \mu\right]$.

Thus, by taking the Laplace transform of Eq. 5 for $k \geq 2$, we can write

$$
\mu^{v} L\left[P_{k}^{v}(t) ; \mu\right]-\mu^{v-1} P_{k}^{v}(0)=-\lambda_{k} L\left[P_{k}^{v}(t) ; \mu\right]+\sum_{i=1}^{k-1} \lambda_{i} L\left[P_{i}^{v}(t) ; \mu\right] .
$$

Therefore,

$$
\begin{aligned}
\left(\mu^{v}+\lambda_{k}\right) L\left[P_{k}^{v}(t) ; \mu\right]= & \sum_{i=3}^{k-1} \lambda_{i} \frac{\lambda_{1} \mu^{v-1}}{\left(\mu^{v}+\lambda_{1}\right)\left(\mu^{v}+\lambda_{i}\right)} \sum_{\left(q_{2}, \ldots, q_{i-1}\right) \in A_{i}} \prod_{j=2}^{i-1}\left(\frac{\lambda_{j}}{\mu^{v}+\lambda_{j}}\right)^{q_{j}} \\
& +\lambda_{2} \frac{\lambda_{1} \mu^{v-1}}{\left(\mu^{v}+\lambda_{1}\right)\left(\mu^{v}+\lambda_{2}\right)}+\lambda_{1} \frac{\mu^{v-1}}{\mu^{v}+\lambda_{1}}
\end{aligned}
$$

and

$$
\begin{aligned}
L\left[P_{k}^{v}(t) ; \mu\right]= & \frac{\lambda_{1} \mu^{v-1}}{\left(\mu^{v}+\lambda_{1}\right)\left(\mu^{v}+\lambda_{k}\right)} \\
& \times\left(\sum_{i=3}^{k-1} \frac{\lambda_{i}}{\left(\mu^{v}+\lambda_{i}\right)} \sum_{\left(q_{2}, \ldots, q_{i-1}\right) \in A_{i}} \prod_{j=2}^{i-1}\left(\frac{\lambda_{j}}{\mu^{v}+\lambda_{j}}\right)^{q_{j}}+\frac{\lambda_{2}}{\left(\mu^{v}+\lambda_{2}\right)}+1\right)
\end{aligned}
$$

Note that

$$
\begin{aligned}
& \sum_{i=3}^{k-1} \frac{\lambda_{i}}{\left(\mu^{v}+\lambda_{i}\right)} \sum_{\left(q_{2}, \ldots, q_{i-1}\right) \in A_{i}} \prod_{j=2}^{i-1}\left(\frac{\lambda_{j}}{\mu^{v}+\lambda_{j}}\right)^{q_{j}}+\frac{\lambda_{2}}{\left(\mu^{v}+\lambda_{2}\right)}+1 \\
& \quad=\sum_{\left(q_{2}, \ldots, q_{k-1}\right) \in A_{k}} \prod_{j=2}^{k-1}\left(\frac{\lambda_{j}}{\mu^{v}+\lambda_{j}}\right)^{q_{j}}
\end{aligned}
$$

so that we get formula (7). 
Lemma 2 Let $x \in \mathbb{R}, a_{i} \in \mathbb{R}(i=1, \ldots n)$ and $a_{i} \neq a_{j}$ for $i \neq j$, then

$$
\frac{1}{\prod_{i=1}^{n}\left(x+a_{i}\right)}=\sum_{i=1}^{n} \frac{1}{\prod_{\substack{j=1 \\ j \neq i}}^{n}\left(a_{j}-a_{i}\right)} \times \frac{1}{\left(x+a_{i}\right)}
$$

Proof We prove Eq. 9 by induction.

For $n=2$, it is clear. We suppose that it is true for $n=k-1$, then we will show that it is true for $n=k$.

$$
\begin{aligned}
& \frac{1}{\prod_{i=1}^{k}\left(x+a_{i}\right)}=\frac{1}{\left(x+a_{k}\right) \times \prod_{i=1}^{k-1}\left(x+a_{i}\right)}=\frac{1}{\left(x+a_{k}\right)} \times \sum_{i=1}^{k-1} \frac{1}{\prod_{\substack{j=1 \\
j \neq i}}^{k-1}\left(a_{j}-a_{i}\right)} \times \frac{1}{\left(x+a_{i}\right)} \\
&=\sum_{i=1}^{k-1} \frac{1}{\prod_{\substack{k=1 \\
j \neq 1}}^{k-1}\left(a_{j}-a_{i}\right)} \times\left(\frac{1}{\left(a_{k}-a_{i}\right)} \times \frac{1}{\left(x+a_{i}\right)}+\frac{1}{\left(a_{i}-a_{k}\right)} \times \frac{1}{\left(x+a_{k}\right)}\right) \\
&=\sum_{i=1}^{k} \frac{1}{\prod_{j=1}^{k}\left(a_{j}-a_{i}\right)} \times \frac{1}{\left(x+a_{i}\right)} . \\
& j \neq i
\end{aligned}
$$

In the next theorem, we will obtain the exact solutions to Eqs. 1 and 2, for $v \in(0,1]$ which will be expressed in terms of finite sums of the Mittag-Leffler function, i.e.

$$
E_{\alpha, \beta}(x)=\sum_{j=0}^{\infty} \frac{x^{j}}{\Gamma(\alpha j+\beta)}, \quad x \in \mathbb{R}, \quad \alpha, \beta \in \mathbb{C}, \quad \Re(\alpha), \Re(\beta)>0 .
$$

Theorem 3 The solution to the problem (1)-(2) for $v \in(0,1]$, is given by

$$
P_{k}^{v}(t)=\left\{\begin{array}{cc}
E_{v, 1}\left(-\lambda_{1} t^{\nu}\right), & k=1 \\
\sum_{j=1}^{k}\left(\sum_{\substack{\left(q_{1}, q_{2}, \ldots, q_{k}\right) \in \tilde{A}_{k} \\
q_{j}}}\left(\prod_{r=1}^{k-1}\left(\lambda_{r}\right)^{q_{r}}\right) \times\left(\prod_{\substack{i=1 \\
i \neq j}}^{k} \frac{1}{\left(\lambda_{i}-\lambda_{j}\right)^{q_{i}}}\right)\right) E_{v, 1}\left(-\lambda_{j} t^{\nu}\right), k=2,3, \ldots
\end{array},\right.
$$

where

$$
\tilde{A}_{k}=\left\{\left(q_{1}, q_{2}, \ldots, q_{k}\right) \mid\left(q_{2}, \ldots, q_{k-1}\right) \in A_{k} \quad \text { and } \quad q_{1}=q_{k}=1\right\}
$$


Proof For $k=1$, by Theorem 1, taking the inverse of Laplace transform and by noting that

$$
L\left[\mathrm{E}_{v, 1}\left(-\lambda t^{v}\right) ; \mu\right]=\frac{\mu^{v-1}}{\left(\mu^{v}+\lambda\right)}
$$

we have

$$
P_{1}^{v}(t)=\mathrm{E}_{v, 1}\left(-\lambda_{1} t^{v}\right)
$$

For $k=2$, from Theorem 1 ,

$$
L\left[P_{2}^{v}(t) ; \mu\right]=\frac{\lambda_{1} \mu^{v-1}}{\left(\mu^{v}+\lambda_{1}\right)\left(\mu^{v}+\lambda_{2}\right)} .
$$

By using Lemma 2, we can get

$$
\begin{aligned}
L\left[P_{2}^{v}(t) ; \mu\right] & =\lambda_{1} \mu^{v-1}\left(\frac{1}{\left(\lambda_{2}-\lambda_{1}\right)} \times \frac{1}{\left(\mu^{v}+\lambda_{1}\right)}+\frac{1}{\left(\lambda_{1}-\lambda_{2}\right)} \times \frac{1}{\left(\mu^{v}+\lambda_{2}\right)}\right) \\
& =\frac{\lambda_{1}}{\left(\lambda_{2}-\lambda_{1}\right)}\left(\frac{\mu^{v-1}}{\mu^{v}+\lambda_{1}}-\frac{\mu^{v-1}}{\mu^{v}+\lambda_{2}}\right) .
\end{aligned}
$$

By taking the inverse of Laplace transform and considering Eq. 12, we obtain

$$
P_{2}^{v}(t)=\frac{\lambda_{1}}{\left(\lambda_{2}-\lambda_{1}\right)}\left(\mathrm{E}_{v, 1}\left(-\lambda_{1} t^{v}\right)-\mathrm{E}_{v, 1}\left(-\lambda_{2} t^{v}\right)\right) .
$$

Then, Eq. 10 is proved for $k=2$.

For $k \geq 3$, from Theorem 1 , we have

$$
L\left[P_{k}^{v}(t) ; \mu\right]=\frac{\lambda_{1} \mu^{v-1}}{\left(\mu^{v}+\lambda_{1}\right)\left(\mu^{v}+\lambda_{k}\right)} \sum_{\left(q_{2}, \ldots, q_{k-1}\right) \in A_{k}} \prod_{j=2}^{k-1}\left(\frac{\lambda_{j}}{\mu^{v}+\lambda_{j}}\right)^{q_{j}} .
$$

By definitions of the sets $A_{k}$ and $\tilde{A}_{k}$ in Eqs. 8 and 11, respectively, we can write

$$
L\left[P_{k}^{v}(t) ; \mu\right]=\mu^{v-1} \sum_{\left(q_{1}, \ldots, q_{k}\right) \in \tilde{A}_{k}} \prod_{r=1}^{k-1}\left(\lambda_{r}\right)^{q_{r}}\left(\frac{1}{\prod_{j=1}^{k}\left(\mu^{v}+\lambda_{j}\right)^{q_{j}}}\right) .
$$

On the other hand, by Lemma 2, we have

$$
\frac{1}{\prod_{j=1}^{k}\left(\mu^{v}+\lambda_{j}\right)^{q_{j}}}=\sum_{j=1}^{k} q_{j}\left(\prod_{\substack{i=1 \\ i \neq j}}^{k} \frac{1}{\left(\lambda_{i}-\lambda_{j}\right)^{q_{i}}}\right) \times \frac{1}{\left(\mu^{v}+\lambda_{j}\right)}, \quad \forall\left(q_{1}, \ldots, q_{k}\right) \in \tilde{A}_{k} .
$$

Therefore, we get

$$
L\left[P_{k}^{v}(t) ; \mu\right]=\sum_{\left(q_{1}, \ldots, q_{k}\right) \in \tilde{A}_{k}} \prod_{r=1}^{k-1}\left(\lambda_{r}\right)^{q_{r}} \sum_{j=1}^{k}\left(q_{j}\left(\prod_{\substack{i=1 \\ i \neq j}}^{k} \frac{1}{\left(\lambda_{i}-\lambda_{j}\right)^{q_{i}}}\right) \times \frac{\mu^{v-1}}{\left(\mu^{v}+\lambda_{j}\right)}\right) .
$$


Fig. 1 Plot of $P_{k}^{v}(t)$ for $v=0.9, k=1,2,3$ and $\lambda_{i}=2^{-i}(i \in \mathbb{N})$

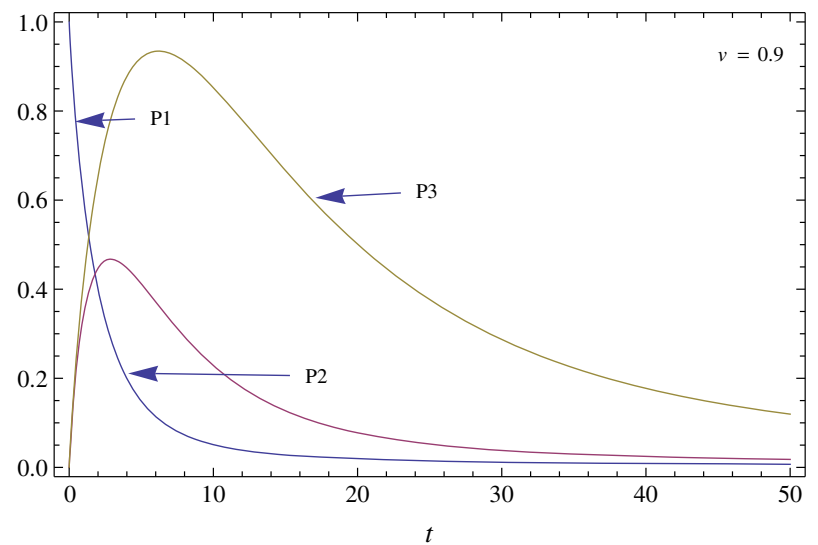

So, by taking the inverse Laplace transform and by Eq. 12, we can obtain

$$
P_{k}^{v}(t)=\sum_{\left(q_{1}, \ldots, q_{k}\right) \in \tilde{A}_{k}} \prod_{r=1}^{k-1}\left(\lambda_{r}\right)^{q_{r}} \sum_{j=1}^{k}\left(q_{j}\left(\prod_{\substack{i=1 \\ i \neq j}}^{k} \frac{1}{\left(\lambda_{i}-\lambda_{j}\right)^{q_{i}}}\right) \times \mathrm{E}_{v, 1}\left(-\lambda_{j} t^{v}\right)\right) .
$$

Finally, by interchanging the two sums, we get formula (10) for $k=2,3, \ldots$

Let $\lambda_{i}=2^{-i}(i \in \mathrm{N})$, we can see the plot of $P_{k}^{0.9}(t)$ for $k=1,2,3$ and $P_{3}^{v}(t)$ for $v=0.6,0.8,1$, in Figs. 1 and 2, respectively.

Remark 1 In formula (10), if we just consider the case $q_{i}=1$ for any $i$, we have

$$
P_{k}^{v}(t)= \begin{cases}\mathrm{E}_{v, 1}\left(-\lambda_{1} t^{v}\right), & k=1 \\ \prod_{r=1}^{k-1}\left(\lambda_{r}\right) \sum_{j=1}^{k}\left(\prod_{\substack{i=1 \\ i \neq j}}^{k} \frac{1}{\left(\lambda_{i}-\lambda_{j}\right)}\right) \mathrm{E}_{v, 1}\left(-\lambda_{j} t^{v}\right), & k=2,3, \ldots\end{cases}
$$

Fig. 2 Plot of $P_{3}^{v}(t)$ for $v=0.6,0.8,1$ and $\lambda_{i}=2^{-i}(i \in \mathbb{N})$

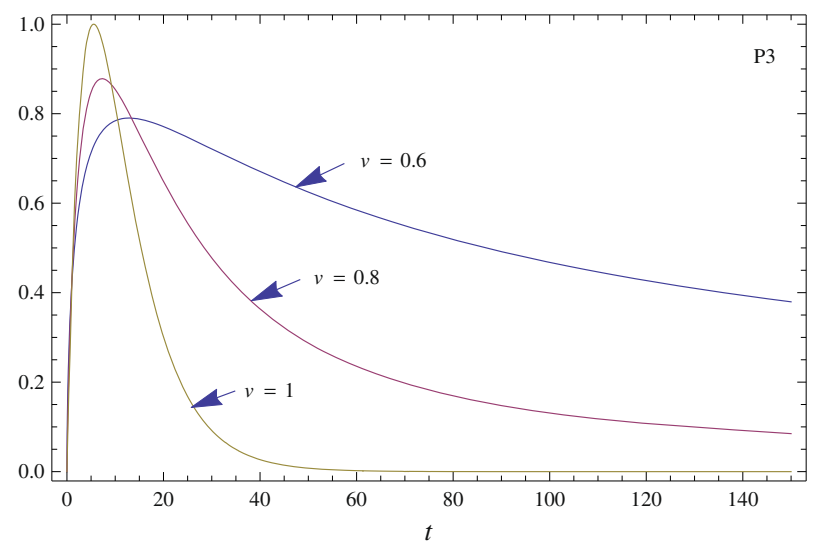


that is the exact solution of the fractional birth equation proposed by Orsingher and Polito (2010).

Remark 2 By considering the special case $v=1$ in Theorem 3 and writing $P_{k}^{1}=P_{k}$, we can conclude that the solution to the integer-order fractional equations

$$
\left\{\begin{array}{l}
\frac{d}{d t} P_{1}(t)=-\lambda_{1} P_{1}(t) \\
\frac{d}{d t} P_{k}(t)=-\lambda_{k} P_{k}(t)+\sum_{i=1}^{k-1} \lambda_{i} P_{i}(t), \quad k=2,3, \ldots,
\end{array} \quad t \geq 0,\right.
$$

with

$$
P_{k}(0)=\left\{\begin{array}{ll}
1 & k=1, \\
0 & k=2,3, \ldots
\end{array} .\right.
$$

is given by

$$
P_{k}(t)=\left\{\begin{array}{cc}
e^{-\lambda_{1} t}, & k=1 \\
\sum_{j=1}^{k}\left(\sum_{\left(q_{1}, q_{2}, \ldots, q_{k}\right) \in \tilde{A}_{k}} q_{j}\left(\prod_{r=1}^{k-1}\left(\lambda_{r}\right)^{q_{r}}\right) \times\left(\prod_{\substack{i=1 \\
i \neq j}}^{k} \frac{1}{\left(\lambda_{i}-\lambda_{j}\right)^{q_{i}}}\right)\right) e^{-\lambda_{j} t}, k=2,3, \ldots .
\end{array}\right.
$$

We prove now that the previous expression represents a proper probability distribution, for any $t \geq 0$, under the condition that $\lambda_{i} \geq 0,(i=1,2, \ldots)$ and thus we can define $\left.P_{k}^{(} t\right)=\operatorname{Pr}\left\{N_{1}(t)=k\right\}$, for the random process $N_{1}(t), t \geq 0$.

Theorem 4 If $\lambda_{i} \geq 0,(i=1,2, \ldots)$, then

(i) $P_{k}(t) \geq 0, t \geq 0, k=1,2, \ldots$

(ii) $\sum_{k=1}^{\infty} \frac{1}{\lambda_{k}}=\infty \Rightarrow \sum_{k=1}^{\infty} P_{k}(t)=1, \forall t \geq 0$.

\section{Proof}

(i) We prove it by induction. For $k=1$ have that $\left.P_{1}^{(} t\right)=e^{-\lambda_{1} t}>0$. For $k=2$, by Eqs. 13 and 14, we get $\frac{d}{d t} P_{2}(t)=-\lambda_{2} P_{2}(t)+\lambda_{1} P_{1}(t)$ and $P_{2}(0)=0$. Therefore

$$
P_{2}(t)=e^{-\lambda_{2} t} \int_{0}^{t} \lambda_{1} e^{\left(\lambda_{2}-\lambda_{1}\right) t} d t \stackrel{\lambda_{1}>0}{\Rightarrow} \quad P_{2}(t) \geq 0 .
$$

If $P_{i}(t) \geq 0$ for $i=1,2, \ldots, k-1$, we show that $P_{k}(t) \geq 0$ as follows.

By solving Eqs. 13 and 14 with respect to $P_{i}(t)(i=1,2, \ldots, k-1)$, we obtain

$$
P_{k}(t)=e^{-\lambda_{k} t} \int_{0}^{t} e^{\lambda_{k} t} \sum_{i=1}^{k-1} \lambda_{i} P_{i}(t) d t \quad \lambda_{i}, P_{i}(t) \geq 0, i=1, \ldots, k-1 \quad P_{k}(t) \geq 0 .
$$

(ii) Let $S_{n}(t)=\sum_{k=1}^{n} P_{k}(t)$ and $S(t)=\sum_{k=1}^{\infty} P_{k}(t)$. Then, from Eq. 14 we have $S_{n}(0)=1$, $\forall n \geq 1$. 
By summing the differential Eq. 13 for $k=1, \ldots, n$ and $n \geq 3$ we have

$$
\begin{aligned}
\sum_{k=1}^{n} \frac{d}{d t} P_{k}(t) & =-\lambda_{n} P_{n}(t)+\sum_{j=3}^{n} \sum_{i=1}^{j-2} \lambda_{i} P_{i}(t) \Rightarrow \frac{d}{d t} S_{n}(t)=-\lambda_{n} P_{n}(t)+\sum_{j=3}^{n} \sum_{i=1}^{j-2} \lambda_{i} P_{i}(t) \\
\int_{0}^{t} \frac{d}{d t} S_{n}(t) d t & =-\lambda_{n} \int_{0}^{t} P_{n}(t) d t+\sum_{j=3}^{n} \sum_{i=1}^{j-2} \lambda_{i} \int_{0}^{t} P_{i}(t) d t \\
S_{n}(t)-1 & =-\lambda_{n} \int_{0}^{t} P_{n}(t) d t+\sum_{j=3}^{n} \sum_{i=1}^{j-2} \lambda_{i} \int_{0}^{t} P_{i}(t) d t .
\end{aligned}
$$

Since $\sum_{j=3}^{n} \sum_{i=1}^{j-2} \lambda_{i} \int_{0}^{t} P_{i}(t) d t \geq 0$, we can write

$$
1-S(t) \leq 1-S_{n}(t) \leq \lambda_{n} \int_{0}^{t} P_{n}(t) d t \leq \lambda_{n} \int_{0}^{+\infty} P_{n}(t) d t, \quad \forall n \geq 2 .
$$

On the other hand, from Eq. 15, we have

$$
\begin{aligned}
& P_{k}(t)=\frac{1}{\lambda_{k}} \sum_{\left(q_{1}, \ldots, q_{k}\right) \in \tilde{A}_{k}} \sum_{j=1}^{k} q_{j} \lambda_{j} \prod_{\substack{i=1 \\
i \neq j}}^{k}\left(\frac{\lambda_{i}}{\lambda_{i}-\lambda_{j}}\right)^{q_{i}} e^{-\lambda_{j} t}, \quad k=2,3, \ldots \\
& \int_{0}^{+\infty} P_{k}(t) d t=\frac{1}{\lambda_{k}} \sum_{\left(q_{1}, \ldots, q_{k}\right) \in \tilde{A}_{k}} \sum_{j=1}^{k} q_{j} \lambda_{j} \prod_{\substack{i=1 \\
i \neq j}}^{k}\left(\frac{\lambda_{i}}{\lambda_{i}-\lambda_{j}}\right)^{q_{i}} \int_{0}^{+\infty} e^{-\lambda_{j} t} d t, \quad k=2,3, \ldots \\
& \int_{0}^{+\infty} P_{k}(t) d t=\frac{1}{\lambda_{k}} \sum_{\left(q_{1}, \ldots, q_{k}\right) \in \tilde{A}_{k}} \sum_{j=1}^{k} q_{j} \prod_{\substack{i=1 \\
i \neq j}}^{k}\left(\frac{\lambda_{i}}{\lambda_{i}-\lambda_{j}}\right)^{q_{i}}, \quad k=2,3, \ldots
\end{aligned}
$$

Moreover, from Eq. 3.12 in Orsingher et al. (2010), we have that

$$
\sum_{\substack { j=1 \\
\begin{subarray}{c}{i=1 \\
i \neq j{ j = 1 \\
\begin{subarray} { c } { i = 1 \\
i \neq j } }\end{subarray}}^{k}\left(\frac{\lambda_{i}}{\lambda_{i}-\lambda_{j}}\right)=1, \quad \forall \lambda_{i}(i=1,2, \ldots, k) \text { and } \lambda_{i} \neq \lambda_{j} \text { for } i \neq j .
$$

Therefore, we can conclude that

$$
\sum_{j=1}^{k} q_{j} \prod_{\substack{i=1 \\ i \neq j}}^{k}\left(\frac{\lambda_{i}}{\lambda_{i}-\lambda_{j}}\right)^{q_{i}}=1, \quad \forall\left(q_{1}, \ldots, q_{k}\right) \in \tilde{A}_{k}
$$


and that

$$
\int_{0}^{+\infty} P_{k}(t) d t=\frac{1}{\lambda_{k}} \sum_{\left(q_{1}, \ldots, q_{k}\right) \in \tilde{A}_{k}} 1=\frac{2^{k-2}}{\lambda_{k}}, \quad k=2,3, \ldots
$$

Thus we can write

$$
\begin{aligned}
1-S(t) & \leq \lambda_{n} \int_{0}^{t} P_{n}(t) d t \leq 2^{n-2}, \quad \forall n \geq 3 \\
& \Rightarrow \frac{(1-S(t))}{\lambda_{n}} \leq \int_{0}^{t} P_{n}(t) d t \leq \frac{2^{n-2}}{\lambda_{n}}, \quad \forall n \geq 3 .
\end{aligned}
$$

Moreover we have that

$$
\int_{0}^{+\infty} P_{1}(t) d t=\frac{1}{\lambda_{1}} \text { and } \quad \int_{0}^{+\infty} P_{2}(t) d t=\frac{1}{\lambda_{2}}
$$

so that we get

$$
\frac{(1-S(t))}{\lambda_{n}} \leq \int_{0}^{t} P_{n}(t) d t \leq \frac{1}{\lambda_{n}}, \quad \forall n=1,2 .
$$

By Eqs. 16, 17 and summing for $n=1, \ldots m, \forall m=3,4, \ldots$, we have

$$
\begin{aligned}
(1-S(t)) \sum_{n=1}^{m} \frac{1}{\lambda_{n}} & \leq \int_{0}^{t} S_{m}(t) d t \leq \frac{1}{\lambda_{1}}+\frac{1}{\lambda_{2}}+\sum_{n=3}^{m} \frac{2^{n-2}}{\lambda_{n}}, \quad \forall m=1,2, \ldots, \\
& \Rightarrow(1-S(t)) \sum_{n=1}^{m} \frac{1}{\lambda_{n}} \leq \int_{0}^{t} S_{m}(t) d t \leq \sum_{n=1}^{m} \frac{2^{n}}{\lambda_{n}}, \quad \forall m=1,2, \ldots
\end{aligned}
$$

Therefore, using the comparison test for series, we have

$$
\sum_{n=1}^{\infty} \frac{2^{n}}{\lambda_{n}}<\infty \Rightarrow \sum_{n=1}^{\infty} \frac{1}{\lambda_{n}}<\infty \quad \text { and } \quad \sum_{n=1}^{\infty} \frac{1}{\lambda_{n}}=\infty \Rightarrow \sum_{n=1}^{\infty} \frac{2^{n}}{\lambda_{n}}=\infty .
$$

Finally, similarly to the proof at page 452 of Feller (1968), we can conclude from Eqs. 18 and 19 that (ii) holds.

Turning back to the fractional case, we prove the following result, which holds for any $v>0$. Let $A^{\alpha}(t)$ be the stable subordinator of index $\alpha$ with parameters $\mu=0, \beta=1$ and $\sigma=\left(t \cos \left(\frac{\pi \alpha}{2}\right)\right)^{1 / \alpha}$, for $t \geq 0$, in the notation of Samorodnitsky and Taqqu (1994); thus its Laplace transform can be written as $L\left[h_{\alpha}(x, t) ; \theta\right]=$ $\exp \left\{-\theta^{\alpha} t \cos \left(\frac{\pi \alpha}{2}\right)\right\}$.

Furthermore its inverse (or hitting time) process is defined as $L^{\alpha}(t)=$ $\inf \left\{z \mid z>0, A^{\alpha}(z)>t\right\}$ for all $t \geq 0$.

Theorem 5 Let $N_{v}(t)$ be the process defined as $N_{1}\left(H_{v}(t)\right), t \geq 0$, where

$$
H_{v}(t)=\left\{\begin{array}{l}
L^{v}(t), \quad v \in(0,1), \\
A^{\frac{1}{v}}(t), v \in(1,+\infty),
\end{array}\right.
$$


and $H_{v}(t)=t$ for $v=1$, under the assumption that $N_{1}$ and $H_{v}$ are independent. Then the distribution $P_{k}^{v}(t)=\operatorname{Pr}\left\{N_{v}(t)=k\right\}, k \geq 1$, satisfies the problem (1)-(2) for any $v>0$.

Proof By definition, we can write

$$
P_{k}^{v}(t)= \begin{cases}\int_{0}^{+\infty} P_{k}(z) l_{v}(z, t) d z, & v \in(0,1], \\ \int_{0}^{+\infty} P_{k}(z) h_{\frac{1}{v}}(z, t) d z, & v \in(1,+\infty),\end{cases}
$$

where $l_{v}(z, t)$ and $h_{\frac{1}{v}}(z, t)$ are the densities of $L^{v}(t)$ and $A^{\frac{1}{v}}(t)$, respectively.

Case $1 v \in(0,1]$ :

We set

$$
a_{j}^{k}= \begin{cases}1 & k=1, j=1, \\ \sum_{\left(q_{1}, \ldots, q_{k}\right) \in \tilde{A}_{k}} q_{j} \prod_{r=1}^{k-1} \lambda_{r}^{q_{r}} \times \prod_{\substack{i=1 \\ i \neq j}}^{k} \frac{1}{\left(\lambda_{i}-\lambda_{j}\right)^{q_{i}}} & k \geq 2, j=1, \ldots, k,,\end{cases}
$$

then formula (10) can be written as $P_{k}^{v}(t)=\sum_{j=1}^{k} a_{j}^{k} E_{v, 1}\left(-\lambda_{j} t^{v}\right)$. Let $G_{v}(u, t)=$ $\sum_{k=1}^{\infty} u^{k} P_{k}^{v}(t)$ be the probability generating function, then we can write

$$
\begin{aligned}
\int_{0}^{+\infty} e^{-\mu t} G_{v}(u, t) d t & =\int_{0}^{+\infty} e^{-\mu t} \sum_{k=1}^{\infty} u^{k} P_{k}^{v}(t) d t \\
& =\int_{0}^{+\infty} e^{-\mu t} \sum_{k=1}^{\infty} u^{k} \sum_{j=}^{k} a_{j}^{k} E_{v, 1}\left(-\lambda_{j} t^{v}\right) d t \\
& =\sum_{k=1}^{\infty} u^{k} \sum_{j=1}^{k} a_{j}^{k} \frac{\mu^{v-1}}{\mu^{v}+\lambda_{j}}=\sum_{k=1}^{\infty} u^{k} \mu^{v-1} \sum_{j=1}^{k} a_{j}^{k} \int_{0}^{+\infty} e^{-s\left(\mu^{v}+\lambda_{j}\right)} d s \\
& =\int_{0}^{+\infty} \mu^{v-1} e^{-s \mu^{v}} \sum_{k=1}^{\infty} u^{k} \sum_{j=1}^{k} a_{j}^{k} e^{-s \lambda_{j}} d s \\
& =\int_{0}^{+\infty} \mu^{v-1} e^{-s \mu^{v}} \sum_{k=1}^{\infty} u^{k} P_{k}(s) d s \\
& =\int_{0}^{+\infty} \mu^{v-1} e^{-s \mu^{v}} G_{1}(u, s) d s \\
& =\int_{0}^{+\infty} e^{-\mu t} \int_{0}^{+\infty} G_{1}(u, s) l_{v}(s, t) d s d t,
\end{aligned}
$$


since $\mu^{v-1} e^{-s \mu^{v}}=\int_{0}^{+\infty} e^{-\mu t} l_{v}(s, t) d t$ (see Hahn et al. 2001). Thus, we get

$$
G_{v}(u, t)=\int_{0}^{+\infty} G_{1}(u, s) l_{v}(s, t) d s \Rightarrow P_{k}^{v}(t)=\int_{0}^{+\infty} P_{k}(s) l_{v}(s, t) d s .
$$

Case $2 v \in(1,+\infty)$ :

For $P_{k}^{v}(t)=\int_{0}^{+\infty} P_{k}(z) h_{\frac{1}{v}}(z, t) d z$, we can write

$$
\begin{aligned}
R L D_{-, t}^{v} P_{k}^{v}(t) & =\int_{0}^{+\infty} P_{k}(z)_{R L} D_{-, t}^{v} h_{\frac{1}{v}}(z, t) d z=\int_{0}^{+\infty} P_{k}(z) \frac{\partial}{\partial z} h_{\frac{1}{v}}(z, t) d z \\
& =\left[P_{k}(z) h_{\frac{1}{v}}(z, t)\right]_{z=0}^{+\infty}-\int_{0}^{+\infty} \frac{d}{d z} P_{k}(z) h_{\frac{1}{v}}(z, t) d z \\
& =-\int_{0}^{+\infty}\left(-\lambda_{k} P_{k}(z)+\sum_{i=1}^{k-1} \lambda_{i} P_{i}(z)\right) h_{\frac{1}{v}}(z, t) d z \\
& \left.\left.=\int_{0}^{+\infty} \lambda_{k} P_{k}^{(} z\right) h_{\frac{1}{v}}(z, t) d z-\sum_{i=1}^{k-1} \lambda_{i} \int_{0}^{+\infty} P_{i}^{(} z\right) h_{\frac{1}{v}}(z, t) d z \\
& =\lambda_{k} P_{k}^{v}(t)-\sum_{i=1}^{k-1} \lambda_{i} P_{i}^{v}(t) .
\end{aligned}
$$

It is well-known that $\lim _{z \rightarrow \infty} h_{\frac{1}{v}}(z, t)=0$ (see Uchaikin and Zolotarev 1999). Moreover the law of $A^{1 / v}(t)$ is governed by the following equation:

$$
{ }_{R L} D_{-, t}^{v} h_{\frac{1}{v}}(z, t)=\frac{\partial}{\partial z} h_{\frac{1}{v}}(z, t), \quad z, t>0, v \in(1,+\infty),
$$

with the following conditions

$$
\left\{\begin{array}{l}
h_{\frac{1}{v}}(0, t)=0 \\
h_{\frac{1}{v}}(z, 0)=\delta(z)
\end{array}\right.
$$

The previous result is proved, for $v=n \in \mathrm{N}$, in D'Ovidio (2011) and later generalized to any $v>1$ in Beghin and Macci (2012) (see formula (5.17), with $\gamma=1 / v$, in the last reference). The proof is complete by considering the definition $\frac{d^{v}}{d t^{v}}:=$ ${ }_{R L} D_{-, t}^{v}, v \in(1,+\infty)$.

Remark 3 The processes representing the random-time arguments in Theorem 5 exhibit different properties, in the two cases $v \in(0,1]$ and $v \in(1,+\infty)$ : the stable subordinator $A^{\frac{1}{v}}(t)$ is strictly increasing and jumps with non-negative probability, while its inverse $L^{v}(t)$ is continuous, non-decreasing and it is not a Lévy process. As a consequence, the sample paths of the two forms of fractional birth processes, i.e. $N_{1}\left(A^{\frac{1}{v}}(t)\right)$ and $N_{1}\left(L^{v}(t)\right)$, display a very different behavior. Indeed in the first case 
the process can perform only unitary jumps in an interval of infinitesimal length (as in the standard case $v=1$ ), while in the latter the number of jumps can be of any integer size.

We are now ready to obtain an explicit expression for the solution to Eqs. 1 and 2 in the case $v \in(1,+\infty)$.

Theorem 6 The solution to problem (1) and (2) is given, for $v \in(1,+\infty)$, by

$$
P_{k}^{v}(t)=\left\{\begin{array}{lr}
e^{-\lambda_{1}^{\frac{1}{v}} t} & k=1, \\
\sum_{j=1}^{k}\left(\sum_{\substack{\left(q_{1}, \ldots, q_{k}\right) \in \tilde{A}_{k} \\
q_{j}}}\left(\prod_{r=1}^{k-1}\left(\lambda_{r}\right)^{q_{r}}\right) \times\left(\prod_{\substack{i=1 \\
i \neq j}}^{k} \frac{1}{\left(\lambda_{i}-\lambda_{j}\right)^{q_{i}}}\right)\right) e^{-\lambda_{j}^{\frac{1}{v}} t} k \geq 2,
\end{array}\right.
$$

Proof From Theorem 5, for $v \in(1,+\infty)$, by considering formula (15), we can get

$$
\begin{aligned}
& P_{k}^{v}(t)=\int_{0}^{+\infty} P_{k}(z) h_{\frac{1}{v}}(z, t) d z \\
& = \begin{cases}\int_{0}^{+\infty} e^{-\lambda_{1} z} h_{\frac{1}{v}}(z, t) d z & k=1, \\
\sum_{j=1}^{k}\left(\sum_{\left(q_{1}, \ldots, q_{k}\right) \in \tilde{A}_{k}} q_{j}\left(\prod_{r=1}^{k-1}\left(\lambda_{r}\right)^{q_{r}}\right) \times\left(\prod_{\substack{i=1 \\
i \neq j}}^{k} \frac{1}{\left(\lambda_{i}-\lambda_{j}\right)^{q_{i}}}\right)\right) \int_{0}^{+\infty} e^{-\lambda_{j} z} h_{\frac{1}{v}}(z, t) d z k \geq 2,\end{cases} \\
& = \begin{cases}e^{-\lambda_{1}^{\frac{1}{v}} t} & k=1, \\
\sum_{j=1}^{k}\left(\sum_{\left(q_{1}, \ldots, q_{k}\right) \in \tilde{A}_{k}} q_{j}\left(\prod_{r=1}^{k-1}\left(\lambda_{r}\right)^{q_{r}}\right) \times\left(\prod_{\substack{i=1 \\
i \neq j}}^{k} \frac{1}{\left(\lambda_{i}-\lambda_{j}\right)^{q_{i}}}\right)\right) e^{-\lambda_{j}^{\frac{1}{v}} t} & k \geq 2,\end{cases}
\end{aligned}
$$

since $\int_{0}^{+\infty} e^{-\lambda_{j} z} h_{\frac{1}{v}}(z, t) d z=e^{-\lambda_{j}^{\frac{1}{v}} t}$ (see Proposition 1.2.12 in Samorodnitsky and Taqqu 1994).

Fig. 3 Plot of $P_{k}^{v}(t)$ for $v=2.75, k=1,2,3$ and $\lambda_{i}=2^{-i}(i \in \mathbb{N})$

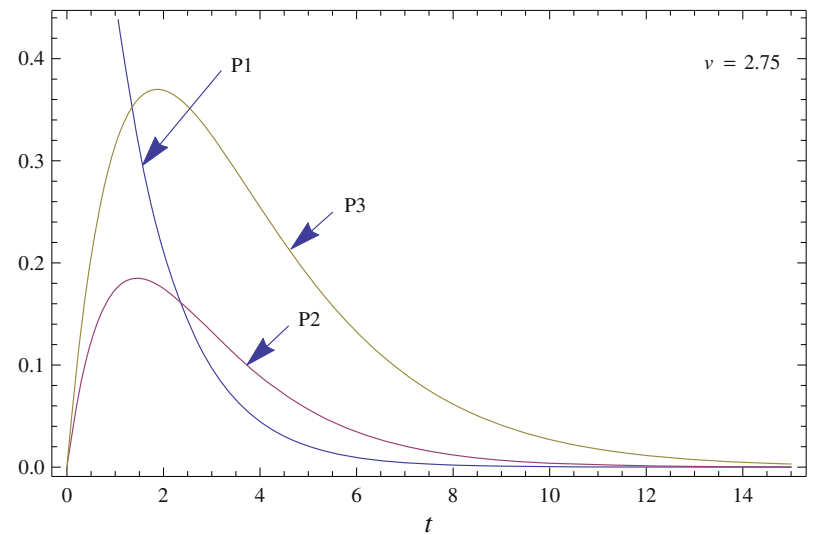


Fig. 4 Plot of $P_{3}^{v}(t)$ for $v=1$, $1.5,1.75$ and $\lambda_{i}=2^{-i}(i \in \mathbb{N})$

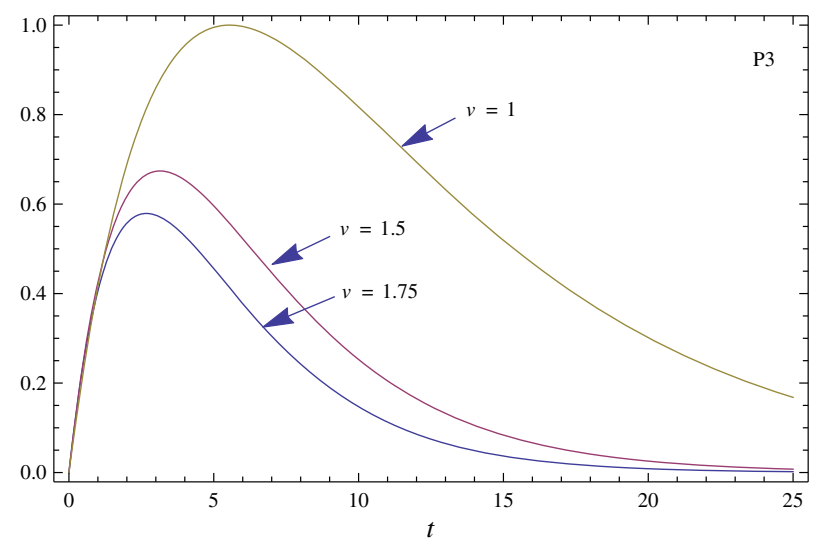

In order to check that the initial conditions in Eq. 2 are satisfied we write:

$$
\begin{aligned}
P_{k}^{v}(0) & =\int_{0}^{+\infty} P_{k}(z) h_{\frac{1}{v}}(z, 0) d z \\
& =P_{1}^{k}(0)=\left\{\begin{array}{ll}
1, & k=1 \\
0, & k=2,3, \ldots
\end{array},\right.
\end{aligned}
$$

since it is well-known that $h_{\frac{1}{v}}(z, 0)=\delta(z)$.

Let $\lambda_{i}=2^{-i}(i \in \mathbb{N})$, then we can see the behavior of $P_{k}^{2.75}(t)$ for $k=1,2,3$ and $P_{3}^{v}(t)$ for $v=1,1.5,1.75$, in Figs. 3 and 4, respectively.

We check now the conditions under which the solution to problem (1) and (2) represents a proper probability distribution.

Corollary 7 The following implications hold:

$$
\begin{array}{cl}
P_{k}(t) \geq 0 \forall k \geq 1, t>0 \quad & \Rightarrow \quad P_{k}^{v}(t) \geq 0 \forall k \geq 1, t>0, v>0, \\
\sum_{k=1}^{\infty} P_{k}(t)=1 \forall t>0 & \Rightarrow \quad \sum_{k=1}^{\infty} P_{k}^{v}(t)=1 \forall t>0, v>0,
\end{array}
$$

where $P_{k}(t)=\operatorname{Pr}\left\{N_{1}(t)=k\right\} \forall k \geq 1, t>0$.

Proof It easily follows from Theorem 5.

Corollary 8 If $\lambda_{i} \geq 0,(i=1,2, \ldots)$, then

(i) $P_{k}^{v}(t) \geq 0, t \geq 0, k=1,2, \ldots$.

(ii) $\sum_{k=1}^{\infty} \frac{1}{\lambda_{k}}=\infty \Rightarrow \sum_{k=1}^{\infty} P_{k}^{v}(t)=1, \forall t \geq 0$.

Proof It follows immediately from Corollary 7 and Theorem 4. 
Acknowledgements The authors thank the associate editor and the referees for the accurate reading of the manuscript and the useful suggestions.

\section{References}

Angulo JM, Ruiz-Medina MD, Anh VV, Greckosch W (2000) Fractional diffusion and fractional heat equation. Adv Appl Probab 32:1077-1099

Beghin L (2012) Fractional relaxation equations and Brownian crossing probabilities of a random boundary. Adv Appl Probab 44:479-505

Beghin L, Macci C (2012) Alternative forms of compound fractional Poisson processes. Abstr Appl Anal 2012:1-30

Beghin L, Orsingher E (2009) Fractional Poisson processes and related planar random motions. Electron J Probab 14(61):1790-1826

Cahoy DO, Polito F (2012) Simulation and estimation for the fractional Yule process. Methodol Comput Appl Probab 14:383-403

D'Ovidio M (2011) On the fractional counterpart of the higher-order equations. Stat Probab Lett 81:1929-1939

Feller W (1968) An introduction to probability theory and its applications, vol 1, 3rd edn. Wiley, New York

Hahn MG, Kobayashi K, Umarov S (2001) Fokker-Planck-Kolmogorov equations associated with time-changed fractional Brownian motion. Proc Am Math Soc 139(2):691-705

Kilbas AA, Srivastava HM, Trujillo JJ (2006) Theory and applications of fractional differential equations. In: North-Holland mathematics studies, vol 204. Elsevier Science B.V., Amsterdam

Mainardi F (1996) The fundamental solutions for the fractional diffusion-wave equation. Appl Math Lett 9(6):23-28

Mainardi F, Gorenflo R, Scalas E (2004) A fractional generalization of the Poisson processes. Vietnam J Math 32:53-64

Meerschaert MM, Nane E, Veillaisamy P (2011) The fractional Poisson process and the inverse stable subordinator. Electron J Probab 59:1600-1620

Orsingher E, Beghin L (2009) Fractional diffusion equations and processes with randomly-varying time. Ann Probab 37(1):206-249

Orsingher E, Polito F (2010) Fractional pure birth processes. Bernoulli 16(3):858-881

Orsingher E, Polito F (2011) On a fractional linear birth-death process. Bernoulli 17(1):114-137

Orsingher E, Polito F (2013) Randomly stopped nonlinear fractional birth processes. Stoch Anal Appl 31:262-292

Orsingher E, Polito F, Sakhno L (2010) Fractional non-linear, linear and sublinear death processes. J Stat Phys 141(1):68-93

Samorodnitsky G, Taqqu MS (1994) Stable non-Gaussian random processes. Chapman Hall, New York

Uchaikin VV (2002) A simple stochastic model for fractional relaxation processes. In: Proceedings of the seminar on stability problems for stochastic models, part I. J Math Sci, vol 111, issue 3. New York, pp 3613-3622

Uchaikin VV, Zolotarev VM (1999) Chance and stability: stable distributions and their applications. VSP, Utrecht 